\title{
Um Objeto de Aprendizagem para apoio a Aprendizagem de Física
}

\author{
Maria de Fátima Webber do Prado Lima, Universidade de Caxias do Sul - \\ mfwplima@ucs.br \\ Douglas Hecher, Universidade de Caxias do Sul - douglashecher@gmail.com \\ Elisa Boff, Universidade de Caxias do Sul - eboff@ucs.br
}

\begin{abstract}
Resumo. A inserção das tecnologias no contexto educacional vem apoiando a aprendizagem de teorias consideradas complexas, tais como as que envolvem matemática aplicada. A possibilidade de interação com o objeto de conhecimento através de modelos e animações explora o imaginário dos estudantes e estimula a aprendizagem lúdica até de temas considerados mais complexos. Um assunto controverso e de difícil assimilação na Física é a "viagem no tempo". Atualmente, sabe-se que viagens para o futuro, baseadas na Teoria da Relatividade Restrita de Albert Einstein, são possíveis, graças a estrutura do Universo. Este artigo explora esta temática e apresenta o processo de desenvolvimento de um OA para aprendizagem de física usando uma abordagem centrada no usuário (aluno), considerando aspectos como estilos de aprendizagem e heurísticas de usabilidade de interface.
\end{abstract}

Palavras chave: Objetos de aprendizagem, Estilos de aprendizagem, Teoria da Relatividade

\section{A Learning Object to support Physics'Learning}

\begin{abstract}
The use of technologies in educational context supports the learning of considered complex theories, such as the ones that involve applied mathematics. Interacting with the knowledge object, through models and animations, explores the imaginary of the students and stimulates the playful learning of subjects even considered complex. A controversial and difficult subject in Physics is the "Time Travel". Currently, it is known that future travel, based in the Theory of the Restricted Relativity of Albert Einstein, are possible, according to structure of the Universe. This paper explores this theme and presents a Learning Object's design process for Physics using a user-centered approach (student), taking into account aspects such as learning styles and interface usability heuristics.
\end{abstract}

Keywords: Learning Objects, Learning Styles, Theory of Relativity

\section{Introdução}

Já é conhecida pela comunidade acadêmica a importância da utilização de ferramentas computacionais para áreas que necessitam cálculos complexos ou simulações avançadas de teorias científicas. Porém, na última década ganhou bastante força a preocupação com os elementos de interação destas ferramentas, com base em teorias de interação e 
aprendizagem promovida no meio social (Vygostky, 1984) (Piaget, 1973) e (Maturana, 1995).

Os Objetos de Aprendizagem (OA) vem sendo utilizados em várias áreas do conhecimento, assim como podemos ver no repositório mantido pelo Australian Learning and Teaching Council ${ }^{1}$ com uma variedade de vídeos sobre Física, incluindo material sobre Energia, Massa, Movimento, Gravidade, Aceleração, entre outros.

Através de pesquisa em repositórios, podemos recuperar um grande número de OAs, com diferentes preocupações em relação a metodologia de desenvolvimento, interface, funcionalidades, aspectos técnicos, formas de apresentação de conteúdo e de promoção da aprendizagem. Estes dois últimos aspectos valem uma atenção mais cuidadosa, visto que as características individuais do aluno, como personalidade, estado afetivo e estilo de aprendizagem, determinam a forma como cada um aprende conceitos, estabelece relações e desenvolve habilidades. Desta forma, a aprendizagem é um processo que envolve modificações estruturais no indivíduo baseado nas suas características individuais e nas suas relações sociais.

A forma particular como uma pessoa adquire conhecimento ou habilidades define seu estilo de aprendizagem. Os estilos de aprendizagem são considerados uma dimensão bipolar e se referem a um modo preferencial em abordar o conteúdo da aprendizagem. Não são "bons" ou "ruins", simplesmente expressam uma tendência (Lopes, 2002).

$\mathrm{Na}$ literatura encontram-se várias formas de classificação dos estilos de aprendizagem, como de Schmeck (1988), Kolb (1985), Myers-Briggs (MBTI) e GrashaRiechman $(1972,1974)$. Neste trabalho, foi adotada a classificação proposta por Felder e Soloman (2010), que apresenta 4 pares de estilos de aprendizagem:

Aprendizes Ativos e Reflexivos - Aprendizes ativos tendem a reter e entender melhor a informação quando fazem algo ativo com a informação, ou seja, quando a discutem ou a explicam para outras pessoas. Já aprendizes reflexivos preferem primeiro pensar sobre a informação que receberam.

Aprendizes Sensitivos e Intuitivos - Aprendizes sensitivos tendem a aprender mais com fatos, enquanto os intuitivos preferem descobrir possibilidades e relações. Sensitivos geralmente resolvem problemas por métodos conhecidos e não gostam de surpresas, já os intuitivos preferem inovação e não gostam de repetição.

Aprendizes Visuais e Verbais - Aprendizes visuais lembram-se melhor do que veem (imagens, diagramas, gráficos, linhas do tempo, filmes e demonstrações). Aprendizes verbais retêm palavras (escritas ou faladas). Mas os aprendizes, em geral, aprendem mais quando a informação é apresentada de ambas as formas, visual e verbal. Para isso, precisam ter a habilidade de processar a informação de ambas as formas. Porém, Felder (2010) observa que a maioria das pessoas são visuais.

Aprendizes Sequenciais e Globais - Aprendizes sequenciais tendem a aprender de forma linear, passo-a-passo, com uma sequência lógica definida. Aprendizes globais tendem a absorver os conteúdos de forma aleatória, sem se preocupar com as conexões, demonstrando um comportamento mais "hipertextual".

Segundo Felder (1998), os ambientes que suportam o processo de ensinoaprendizagem devem incluir momentos variados para atender alunos de diferentes estilos. Assim, os cursos podem incluir momentos onde haja apresentação de conceitos 
apresentando os conteúdos estruturantes (global); possa balancear teoria e modelos (intuitivo) com demonstrações e exemplos (sensorial); utilize gráficos, gravuras, diagramas, esboços (visual) para suplementar a informação (verbal); inclua números, bem como exemplos algébricos (sensorial, indutivo) para ilustrar conceitos abstratos (intuitivo, dedutivo); propicie momentos para uma participação (ativo) e reflexão do material apresentado (reflexivo).

O projeto de um software educativo deve prever em sua fase de análise requisitos relacionados a aprendizagem do aluno. Neste trabalho, o conteúdo apresentado no Objeto de Aprendizagem "Janelas do Tempo" foi elaborado de forma a contemplar alunos com estilos de aprendizagem ativos, reflexivos, visuais, verbais, sequenciais e globais.

Na seção 2 serão apresentados os conceitos relacionados a OA. Na seção 3 será apresentado o projeto e implementação do OA "Janelas do Tempo". Por fim, são apresentadas as considerações finais.

\section{Objetos de Aprendizagem}

A rápida evolução computacional permitiu que novas tecnologias fossem inseridas neste contexto de aprendizagem. Atualmente um dos recursos digitais bastante utilizados são os Objetos de Aprendizagem (OA). Sosteric (2002) mostra que existe uma certa confusão na definição formal: "Objetos de Aprendizagem são coisas diferentes para diferentes profissionais de ensino. De fato, parece haver tantas definições como pessoas a definir". Já o comitê LTSC (Learning Technology Standards Committee), da IEEE ${ }^{2}$, apresenta uma definição largamente utilizada por desenvolvedores de OAs, a saber: "qualquer entidade, digital ou não digital, que pode ser usada, re-utilizados ou referenciada durante o aprendizado apoiado tecnologia".

Na construção de objetos de aprendizagem destacam-se dois padrões: o SCORM (desenvolvido pela ADL - Advanced Distributed Learning Iniciative) e o IMS Learning Design (criado pela Instructional Management Systems Project). O padrão SCORM possui como foco principal a interação individual que o aprendiz possui com o conteúdo do Objeto de Aprendizagem, enquanto que o IMS se dedica a padronizar e avaliar toda a atividade de aprendizagem.

O Sharable Content Object Reference Model (SCORM) é um conjunto unificado de especificações para a disponibilização de conteúdo e serviços do tipo $e$ learning, ou seja, de ensino através da internet. Este conjunto possui especificações para a agregação do conteúdo, modelos de sequenciamento e o ambiente de execução.

A principal proposta do Modelo SCORM é definir uma sistemática de interação entre os conteúdos de aprendizagem e os sistemas de gestão de aprendizagem, ambos sob certificação da ADL, proporcionando independência da plataforma em que está sendo utilizado e possibilitando a migração de Objetos de Aprendizagem que estejam armazenados em ambientes diferentes de forma simples.

\section{Objetos de Aprendizagem no Ensino de Física}

Para Young (2003), um modelo físico é uma representação simplificada de algo considerado complexo demais para ser analisado em sua totalidade, e a construção de um Objeto de Aprendizagem permite justamente a simulação de fenômenos Físicos e a exploração de conceitos relacionados a eles. Porém, como ressalta Dag (2009), a aprendizagem utilizando tecnologias computacionais apesar de ser atrativa é complexa, 
pois deve contemplar as diferentes características de aprendizado. Desta forma, o projeto de um OA deve considerar que os alunos apresentam diferentes estilos cognitivos. Por exemplo, para um aprendiz ativo, devem ser propostos exercícios; para um aprendiz reflexivo, devem ser fornecidos exemplos onde ele possa observar para aprender; para aprendizes globais, a aprendizagem não pode ser proposta de forma incremental (Graf, 2009).

Analisando alguns OAs disponíveis na área da física, pode-se perceber a complexidade de considerar os diferentes tipos de aprendizagem dos alunos. Por exemplo, os objetos de física disponíveis no repositório RIVED $^{3}$ e no laboratório virtual da USP $^{4}$ disponibilizam OAs que apresentam a teoria envolvida de forma visual e verbal, mesclando a teoria com propostas de atividades que devem ser realizadas. Porém, os objetos são construídos de forma linear, obrigando o aluno a passar por todas as etapas propostas. Já os OAs do NTNUJAVA Virtual Physics Laboratory ${ }^{5}$ basicamente são constituídos por páginas HTML que apresentam em forma de textos curtos a explicação teórica do processo físico. Estas páginas contém chamadas a programas desenvolvidos em linguagem Java que simulam alguns parâmetros envolvidos no processo físico.

\section{Objeto de Aprendizagem "Janelas do Tempo"}

O OA "Janelas do Tempo" tem como objetivo apresentar os conceitos físicos envolvidos na teoria da relatividade restrita. Este objeto se utiliza de máquinas do tempo criadas na ficção científica, tais como o Delorean (dos Filmes De Volta para o Futuro) e as Aves de Rapina Klingon (de Jornada nas Estrelas), para facilitar o entendimento das leis da teoria da relatividade. O objeto permite que o usuário simule a dilatação espaço-temporal prevista na Teoria da Relatividade.

O processo de desenvolvimento do OA seguiu uma metodologia adaptada dos trabalhos propostos por Oliveira (2001) e Campos (1995), os quais, por sua vez, foram baseados em modelos de ciclo de vida da área de Engenharia de Software, como o de prototipação e iterativo. O processo seguiu as etapas de:

- Pesquisa de campo com especialistas da área do conhecimento para identificar as melhores formas de se trabalhar o conteúdo;

- Identificação do público-alvo e suas características: É importante conhecer o público-alvo a fim de definir as melhores formas de projetar a interface, de selecionar os conteúdos e propor estratégias de aprendizagem;

- Escolha do conteúdo;

- Análise dos conhecimentos prévios necessários. Se a utilização do OA prevê conhecimentos prévios, o próprio OA deve oferecer recursos (help, FAQ, documentos, ...) para suprir possíveis deficiências dos alunos;

- Identificação dos conceitos estruturantes do conteúdo para facilitar o planejamento da apresentação dos conteúdos e das atividades propostas;

- Documentação dos Requisitos (desenvolvimento dos Modelos de Sistema);

- Prototipação (elaboração de um protótipo de interface funcional);

- Refinamento;

- Implementação;

- Desenvolvimento da documentação do Software Educativo (elaboração da ficha técnica do produto e do manual de instruções para o professor e para o aluno);

- Validação (Utilização e avaliação) do Software Educativo. Esta última etapa ainda está em desenvolvimento. 
A Tabela 4 abaixo relaciona os principais requisitos funcionais, identificados pelos códigos RF e não funcionais do artefato, identificados como RNF.

Tabela 4 - Requisitos do Projeto

\begin{tabular}{|l|l|}
\hline Código & Descrição \\
\hline RNF-001 & O Software deve ser intuitivo, de auto-aprendizagem. \\
\hline RNF-002 & Interface gráfica de fácil utilização \\
\hline RF-001 & $\begin{array}{l}\text { Os recursos necessários para sua utilização estarão disponíveis } \\
\text { gratuitamente para download na internet }\end{array}$ \\
\hline RF-002 & Deve ser construído conforme o padrão SCORM. \\
\hline RF-003 & O Software deve contemplar viagem no tempo para o futuro e passado. \\
\hline RF-004 & No caso da Viagem para o Passado, um paradoxo deve ser criado. \\
\hline
\end{tabular}

\subsection{Princípios Utilizados no Desenvolvimento do Objeto}

Como a teoria envolvida neste objeto de aprendizagem é bastante complexa, alguns cuidados foram tomados no decorrer do seu projeto relativos a apresentação dos conteúdos envolvidos, a utilização de materiais multimídia que atraíssem o interesse do usuário e o projeto de interface do objeto.

$\mathrm{Na}$ apresentação dos conteúdos teóricos, o objeto de aprendizagem não se restringiu a apresentar a Teoria da Relatividade de Albert Einstein e a explicação do cálculo efetuado na simulação da viagem do tempo. $O$ objeto apresenta as teorias complementares necessárias para a compreensão do tema, tais como o conceito de quarta dimensão, mecânica quântica e suas infinitas probabilidades, Teoria das Cordas, Teoria $\mathrm{M}$ e as Realidades Paralelas, Universos Paralelos e a Teoria dos Buracos de Minhoca.

Para atrair a atenção do usuário, o objeto utilizou recursos multimídia. A explicação das teorias vinculadas a Teoria da Relatividade foi apresentada através de documentários que mostram gráficos, simulações e animações, permitindo que o usuário, de maneira visual, compreenda os conceitos matemáticos relacionados. $\mathrm{O}$ objeto apresenta também várias máquinas do tempo criadas na ficção científica. Para explicar o funcionamento destas máquinas, foram selecionados trechos dos filmes onde elas aparecem, além de um texto no formato pdf explicando as questões teóricas envolvidas. Além disso, todos os vídeos selecionados são acompanhados por uma trilha sonora que ajuda a prender a atenção do usuário, motivando-o a assistir os vídeos.

Outro aspecto de relevância foi o projeto da interface. Procurou-se construir uma interface capaz de permitir uma interação fácil e agradável de acordo com os princípios de usabilidade (Nielsen, 2010). O objeto foi desenvolvido com 4 abas distintas e ordenadas de forma que o aluno conheça gradativamente o assunto. As primeiras abas propiciam que o aluno conheça as teorias relacionadas ao objeto de aprendizagem e a última aba que ele efetue o cálculo que simula a dilatação espaço-temporal prevista na Teoria da Relatividade. 


\subsection{Funcionalidades do Objeto de Aprendizagem}

A tela de abertura do Objeto de Aprendizagem possibilita o aluno assistir um vídeo introdutório que tem a finalidade de aguçar a curiosidade do aprendiz quanto ao tema proposto. Algumas frases chaves são mostradas no decorrer do vídeo, questionando sobre a veracidade de viagens ao passado e ao futuro.

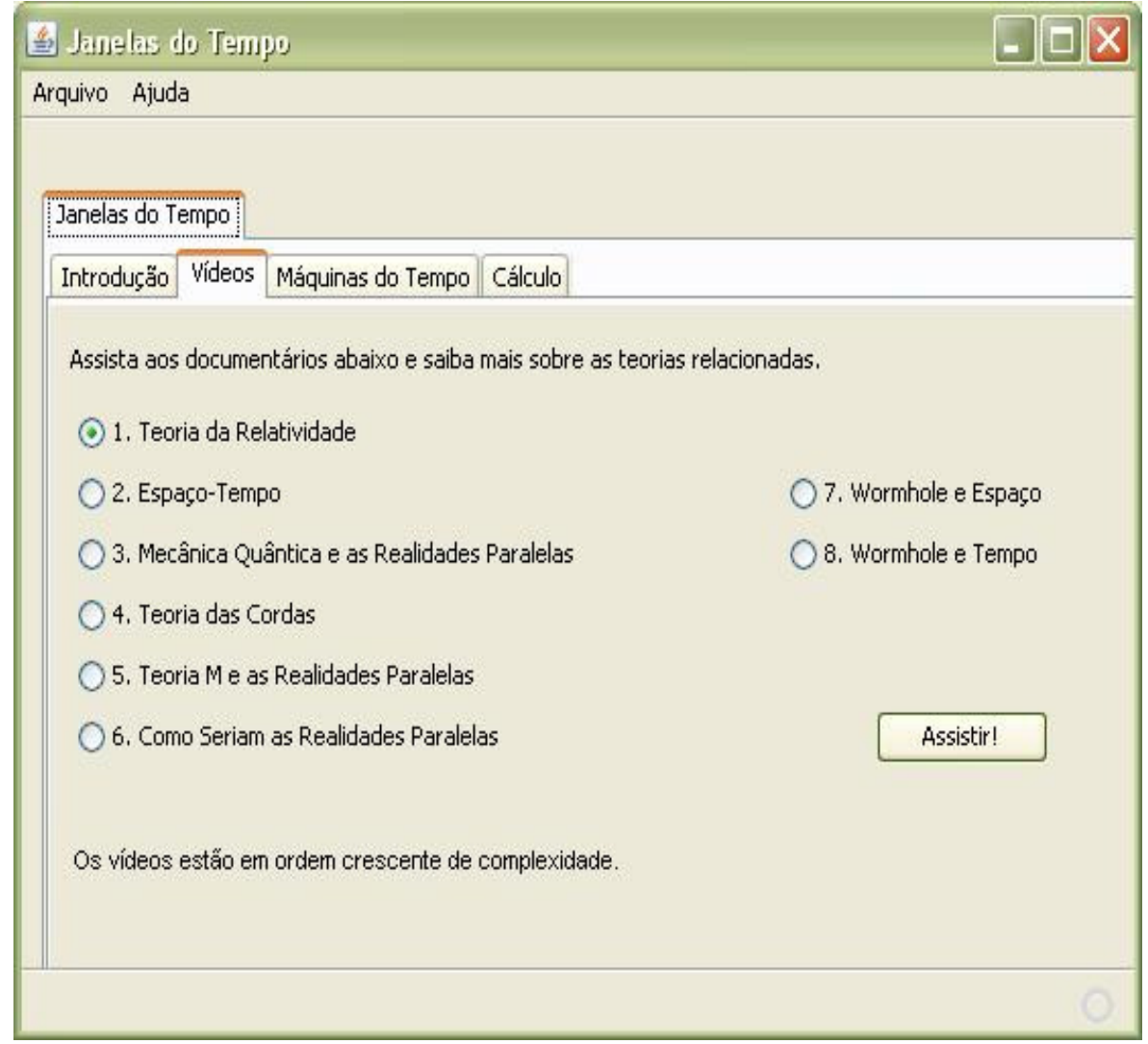

Figura 1: Aba Vídeos

A aba "Vídeos" (Figura 1) apresenta as teorias físicas relacionadas a viagem no tempo. Estas teorias são apresentadas utilizando trechos de documentários. Na interface, os vídeos estão numerados (de um a oito) respeitando a complexidade dos assuntos tratados e sugerindo que estes vídeos sejam assistidos na ordem sequencial. Desta forma, o aluno mesmo desconhecendo qualquer informação sobre as teorias, pode selecioná-los em ordem crescente de complexidade e interação.

A aba "Máquinas do Tempo" (Figura 2) apresenta seis máquinas utilizadas em filmes, buscando familiarizar o usuário com o assunto. Nesta aba, o usuário pode optar em assistir um trecho deste filme ou ler um texto em formato pdf que explica sobre a máquina do tempo, discutindo os conceitos físicos envolvidos nesta máquina. As máquinas do tempo selecionadas foram: Ave de Rapina que resolve a equação da dilatação temporal para velocidades iguais a da luz e explica de que forma o tempo chega a zero e a massa ao infinito; NCC-1701 Enterprise que apresenta o Paradoxo dos Gêmeos; Delorean que trata da teoria dos buracos de minhoca; Máquina do Tempo de H.G. Wells que resolve a equação da dilatação temporal para velocidades acima da luz; Nave do filme Planeta dos Macacos que apresenta o Efeito Doppler para velocidades próximas da luz e o Trem do Dr. Brown que mostra alguns paradoxos temporais criados com as viagens no tempo para o passado. 


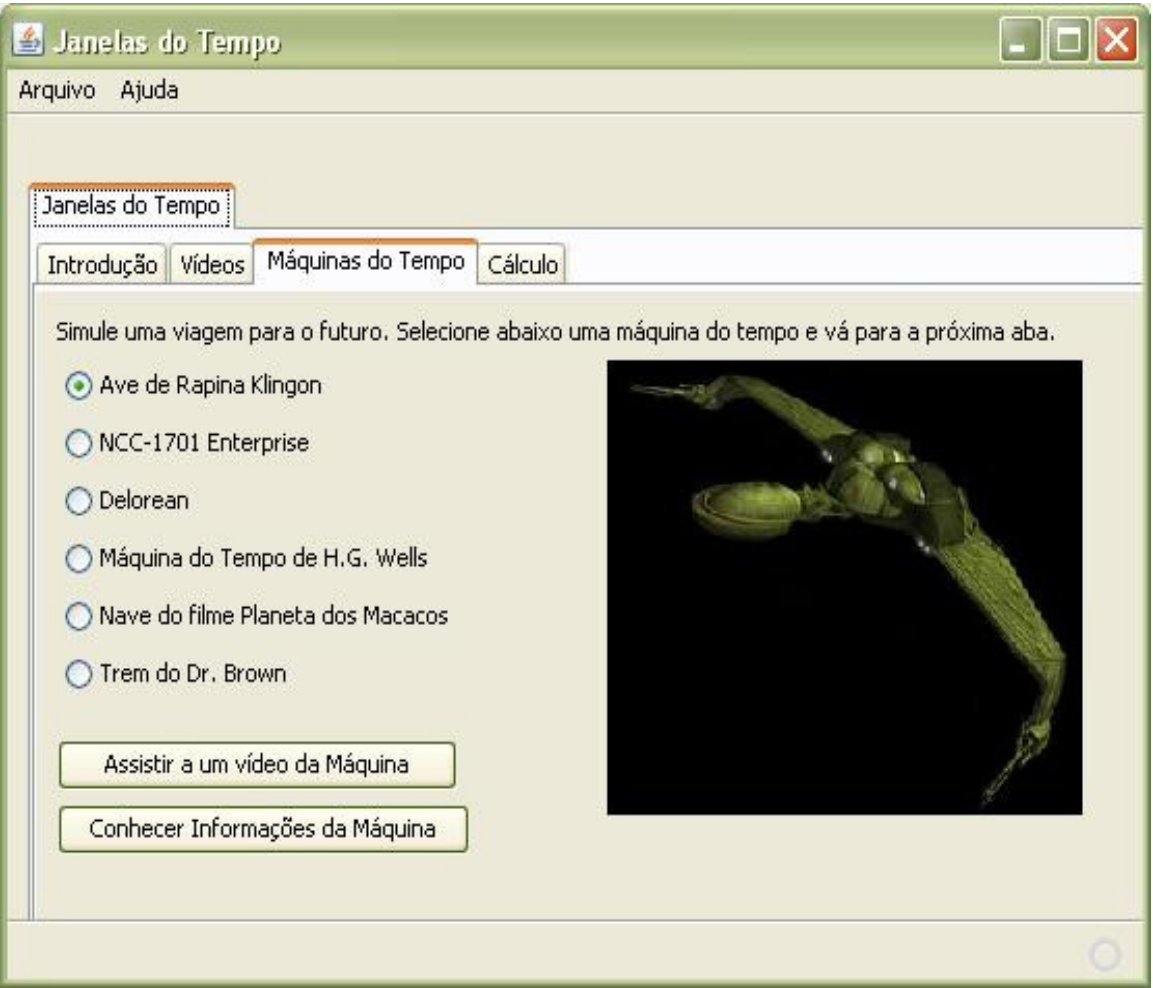

Figura 2: Aba Máquinas do Tempo

A aba "Cálculo" (Figura 3) permite ao usuário simular a dilatação espaçotemporal prevista na Teoria da Relatividade. O campo "Data Atual" traz, de maneira automática, a data do computador onde está sendo executado o objeto.

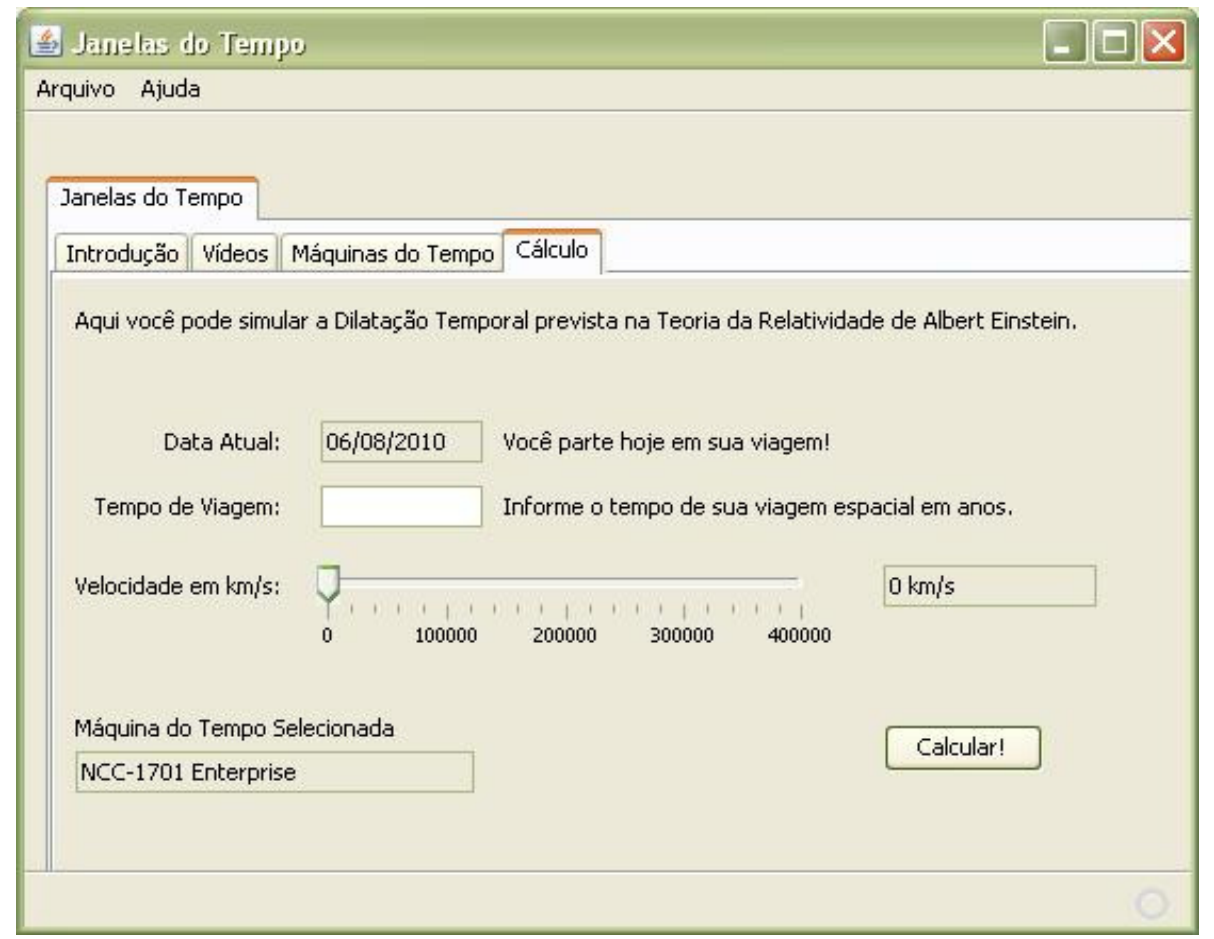

Figura 3: Aba Cálculo

O usuário informa o tempo de viagem em anos e a velocidade em $\mathrm{km} / \mathrm{s}$ utilizando uma barra seletora. $\mathrm{O}$ cálculo é realizado de acordo com a máquina do tempo 
selecionada na aba anterior (aba "Máquinas do Tempo") e seu nome é mostrado abaixo da barra de velocidade. O resultado da equação "t $/ \mathrm{T}=\sqrt{ } 1-(\mathrm{V} / \mathrm{C})^{2}$ " é informado em uma mensagem na tela (Figura 4).

Se o usuário estiver executando o objeto pela primeira vez, ao clicar no botão "OK" da mensagem mostrada ao usuário (Figura 4), um vídeo será apresentado explicando que a viagem no tempo foi cancelada por uma mensagem proveniente de um Universo Paralelo, iniciando um jogo de paradoxos temporais com o usuário, baseado na Mecânica Quântica.

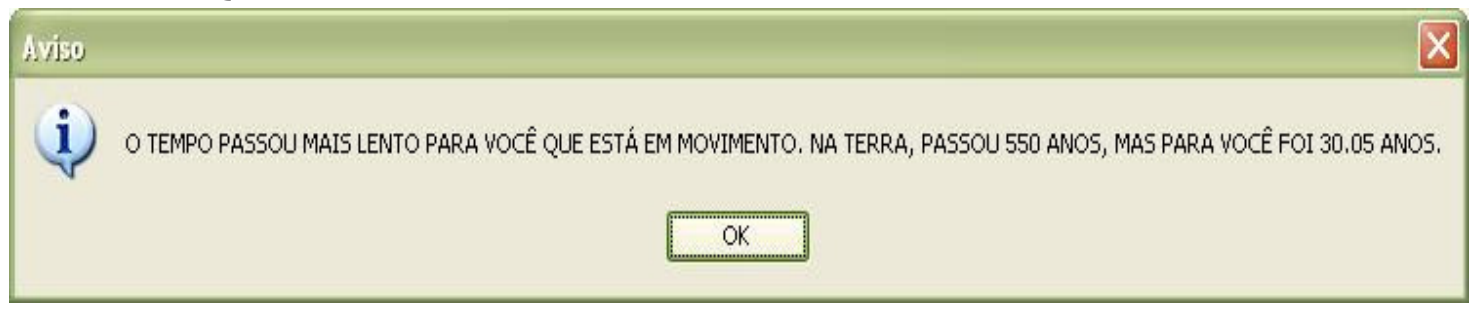

Figura 4: Mensagem ao usuário com o resultado do cálculo

A barra de menus no alto da tela (Figura 3) possui duas opções. Em "Arquivo" existe a opção para fechar o Objeto de Aprendizagem. Em "Ajuda" existem três opções: "Problemas?" que mostra em formato texto, trazendo ao usuário as informações de onde obter os programas para executar os vídeos e o leitor de PDF; "Como Utilizar" que demonstra ao usuário as funções do software e "Sobre" traz os créditos dos vídeos, das músicas e do trabalho.

\subsection{Ferramentas Utilizadas no Desenvolvimento do Objeto de Aprendizagem}

O objeto de aprendizagem foi desenvolvido utilizando a linguagem de de programação Java, utilizando a IDE (Integrated Development Environment) NetBeans ${ }^{6}$. Para realizar o processo de diagramação do sistema foi utilizado UML (Unified Modeling Language).

Todos os vídeos utilizados na construção do objeto foram codificados no formato MPEG2, utilizando dois softwares: o NeroVision Express $3^{7}$ e o Convert Vídeo do software JetAudio ${ }^{8}$. A função principal do JetAudio foi converter alguns vídeos para um formato compatível com o NeroVison, além de inserir as legendas no arquivo. Nenhum vídeo foi utilizado em sua íntegra. Todos passaram pelo processo de conversão e cortes. A versão final foi gerada pelo NeroVision. As músicas foram convertidas utilizando o Audacity'.

Um arquivo de legenda foi criado para o vídeo referente a Realidade Paralela. $\mathrm{O}$ software utilizado foi o Subtitle Workshop $4^{10}$. Após ter sido criado o arquivo, de extensão .SRT, ele foi inserido, juntamente com o vídeo, no programa Convert Vídeo do software JetAudio, para que ambos se tornassem um único arquivo. Sua conversão final foi através do NeroVision.

Os filmes foram codificados através de mídias originais, extraindo-se os arquivos com extensão .VOB e convertendo-os com o auxílio do NeroVision. Já os documentários foram encontrados na internet, em sites como o YouTube ${ }^{11} \mathrm{e}$ posteriormente editados, mantendo os direitos reservados a seus produtores $\mathrm{e}$ idealizadores.

Para converter o objeto de aprendizagem no padrão SCORM foi utilizado o software Trident ${ }^{12}$. Ao transformar o código em um objeto no padrão SCORM, houve 
um cuidado nas informações cadastradas no software no que se refere ao manifesto, para que as informações técnicas e pedagógicas cadastradas facilitassem a busca do OA.

\section{Considerações finais}

Como este OA trata de conteúdos complexos como a teoria da relatividade e a mecânica quântica, foi necessário projetar um objeto que atraísse a atenção do aluno. A utilização de filmes de ficção científica tem o intuito de prender a atenção e ao mesmo tempo despertar a curiosidade dos aprendizes se as situações mostradas são apenas ficção ou se algum dia elas poderão ocorrer. Para terem estas respostas, os alunos obrigatoriamente terão que conhecer os conceitos físicos envolvidos. A trilha sonora que acompanha os vídeos também foi selecionada com a perspectiva de integrar o aprendiz a cena.

No projeto deste OA houve uma preocupação com o processo de desenvolvimento (metodologia apresentada na seção 3), com a observação dos princípios de usabilidade (Nilsen, 2010) no projeto da interface e com a forma de apresentar os conteúdos, a fim de contemplar alunos com estilos de aprendizagem diferentes. A apresentação do conteúdo na forma de textos atende a aprendizes verbais, enquanto os vídeos e animações atendem a aprendizes visuais. Os tópicos de conteúdo foram apresentados na interface em ordem de complexidade, sequencial, o que facilita a aprendizagem de alunos sequenciais. Já alunos com preferência ao estilo global, tem a possibilidade da navegação aleatória nos tópicos de conteúdo. Alunos ativos, em geral, terão o impulso inicial de testar o cálculo da viagem no tempo para depois entenderem o resultado da operação. Já alunos com preferência pelo estilo reflexivo, vão ler o conteúdo, visualizar os vídeos e depois realizar o cálculo da viagem no tempo para comprovar a sua reflexão inicial.

O OA foi transformado para o padrão SCORM pois houve a intenção de desenvolver um objeto de ensino que pudesse ser utilizado em diversas plataformas, possibilitando a migração deste objeto de forma simples. Além disso, este padrão proporciona a criação de objetos com conteúdo reutilizável, permitindo a edição e adaptação do sistema a outras tecnologias existentes, incluindo editores de linguagem XML, HTML e Javascript.

Para permitir uma maior compatibilidade e não perder a qualidade, os vídeos foram gerados no formato MPEG2. Porém, esta decisão acarretou em arquivos de vídeo de tamanho muito grande. Será necessário estudar algumas alternativas para diminuir o tamanho do OA, que passem pela geração do vídeo em outro formato ou a diminuição da qualidade do vídeo sem prejudicar todo o projeto do OA.

\footnotetext{
${ }^{1}$ http://www.physclips.unsw.edu.au/

${ }^{2} \mathrm{http} / / / \mathrm{ltsc}$.ieee.org/wg12/index.html

${ }^{3} \mathrm{http}$ ://www.nce.ufrj.br/ginape/rived/objetos.htm

${ }^{4} \mathrm{http}: / / \mathrm{www}$. labvirt.fe.usp.br/

${ }^{5} \mathrm{http}: / / \mathrm{www} \cdot$ phy.ntnu.edu.tw

${ }^{6} \mathrm{http}: / / \mathrm{www}$. netbeans.com

${ }^{7} \mathrm{http}: / / \mathrm{www}$. nero.com

${ }^{8} \mathrm{http}: / /$ www.cowonamerica.com

${ }^{9} \mathrm{http}: / /$ audacity.sourceforge.net

${ }^{10} \mathrm{http}: / / \mathrm{www}$.urusoft.net

${ }^{11} \mathrm{http}: / / \mathrm{www}$. youtube.com

${ }^{12} \mathrm{http}: / / \mathrm{www}$. scormsoft.com/trident
}

V. $8 \mathrm{~N}^{\mathrm{o}} 3$, dezembro, 2010 


\section{Referências}

Campos, F. C. A.; Campos, G. H. B. de; Rocha, A. R. C. da. (1995) Dez Etapas para o Desenvolvimento de Software Educacional do Tipo Multimídia. Relatório Técnico do Programa de Engenharia de Sistemas e Computação. ES-360/95. Novembro, 1995.

Dag, F.; Geçer, A.(2009) Relations between online learning and learning styles. World Conference on Educacional Sciences 2009, p 862-871.

Felder, R. M. and Silverman, L. K. (1998) Learning Styles and Teaching Styles in Enginnering Education. Engr. Education, v.78, n.7, p.674-681, 1998.

Felder, R. M. and Soloman, B. A. (2010) Learning Styles and Strategies. Disponível na Internet em: http://www4.ncsu.edu/unity/lockers/users/f/felder/public/ILSdir/styles.htm. Acessado em Março de 2010.

Graf, S.; Liu, T.; Yang, S.(2009). Learning styles and cognitive traits - Their relationship and its benefits in web-based educational systems. Computers in Human Behavior, n.25, p 128-1289.

Grasha, A. F.; Riechmann, S. A Rational Approach to Developing and Assessing the Construct Validity of a Student Learning Scale Instrument. The Journal of Psychology, 1974, n.87, p.213-223.

Kolb, D. A. Learning Style Inventary Technical Manual. Boston: Hay McBer, 1985.

Lopes, Wilma Maria Guimarães. (2002) ILS - Inventário de Estilos de Aprendizagem de Felder-Saloman: Investigação de sua Validade em Estudantes Universitários de Belo Horizonte. Dissertação. UFSC.

Maturana, H., Varela, F. (1995) Árvore do conhecimento. Ed. Psy, Campinas, 1995.

Myers, I. B.; Mccaulley, M. H. Manual: a guide to the development and use of the Myers-Briggs type indicador (2nd ed.). Palo Alto: Consulting Psychologist Press, 1986.

Nielsen, J. Heuristic Evaluation. Capturado em Julho de 2010. Online. Disponível na Internet em: http://www.useit.com/papers/heuristic. Julho, 2010.

Oliveira, C. C. de; Costa, J. W. (2001) Ambientes informatizados de aprendizagem: produção e avaliação de software educativo. São Paulo: Papirus, 2001.

Piaget, J. (1973) Estudos Sociológicos. Rio de Janeiro: Forense, 1973. 243p.

Schmeck, R. R. et alli. Learning Strategies and Learning Styles. New York: Plenum Press, 1988.

Sosteric M.; Hesemeier, S. (2002) When is a Learning Object not an Object: A first step towards a theory of learning objects. International Review of Research in Open and Distance Learning. Vol.3, N.2.

Vygotsky, L.S. (1984) A formação social da mente. São Paulo: Martins Fontes, 1984.

Young, H.D. ; Freedaman, R. A.(2003); Sears e Zemansky Física I: Mecânica; tradução e revisão técnica: Adir Moysés Luiz. - $10^{\mathrm{a}}$ ed. - São Paulo: Addison Wesley. 